\title{
Commentary \\ Estimating Copy-Number Proportions: The Comeback of Sanger Sequencing
}

\author{
Eyal Seroussi (1)
}

check for updates

Citation: Seroussi, E. Estimating Copy-Number Proportions: The Comeback of Sanger Sequencing. Genes 2021, 12, 283. https://doi.org /10.3390/genes12020283

Academic Editor: Anelia D. Horvath Received: 31 January 2021

Accepted: 15 February 2021

Published: 17 February 2021

Publisher's Note: MDPI stays neutral with regard to jurisdictional claims in published maps and institutional affiliations.

Copyright: (C) 2021 by the author. Licensee MDPI, Basel, Switzerland. This article is an open access article distributed under the terms and conditions of the Creative Commons Attribution (CC BY) license (https:// creativecommons.org/licenses/by/ $4.0 /)$.
Institute of Animal Science, Agricultural Research Organization (ARO), HaMaccabim Road, P.O.B 15159, Rishon LeTsiyon 7528809, Israel; seroussi@agri.huji.ac.il

\begin{abstract}
Determination of the relative copy numbers of mixed molecular species in nucleic acid samples is often the objective of biological experiments, including Single-Nucleotide Polymorphism (SNP), indel and gene copy-number characterization, and quantification of CRISPR-Cas9 base editing, cytosine methylation, and RNA editing. Standard dye-terminator chromatograms are a widely accessible, cost-effective information source from which copy-number proportions can be inferred. However, the rate of incorporation of dye terminators is dependent on the dye type, the adjacent sequence string, and the secondary structure of the sequenced strand. These variable rates complicate inferences and have driven scientists to resort to complex and costly quantification methods. Because these complex methods introduce their own biases, researchers are rethinking whether rectifying distortions in sequencing trace files and using direct sequencing for quantification will enable comparable accurate assessment. Indeed, recent developments in software tools (e.g., TIDE, ICE, EditR, BEEP and BEAT) indicate that quantification based on direct Sanger sequencing is gaining in scientific acceptance. This commentary reviews the common obstacles in quantification and the latest insights and developments relevant to estimating copy-number proportions based on direct Sanger sequencing, concluding that bidirectional sequencing and sophisticated base calling are the keys to identifying and avoiding sequence distortions.
\end{abstract}

Keywords: dye-terminator DNA sequencing; SNP; CNV; CpG; bisulfite sequencing; base editor; RNA editing; replication diode; heteroplasmy

\section{Introduction}

Incorporation of chain-terminating dideoxy nucleotide analogues (ddNTPs) during in-vitro DNA polymerization is the basis of the Sanger sequencing method, named after its first developer Frederick Sanger [1]. Aiming at higher throughput, identical nucleotide resolution, and better signal strength, accuracy, and read length, further developments to the Sanger protocol have implemented fluorescent dye-labeled ddNTP terminators and engineered thermostable DNA polymerases [2]. Today, Sanger sequencing is commercially dominated by capillary sequencers using BigDye ${ }^{\circledR}$ terminators and Thermus aquaticus (Taq) FS polymerase (AmpliTaq ${ }^{\circledR}$, ThermoFisher Scientific, Waltham, MA, USA [3]). Commercially available as Thermo Sequenase ${ }^{\mathrm{TM}}[4]$, this enzyme is an engineered variant of Taq DNA polymerase that contains two mutations, leading to a much more even peak-intensity pattern. A point mutation (F667Y) in the active site results in less discrimination against ddNTPs. The second amino-terminal mutation eliminates the $5^{\prime} \rightarrow 3^{\prime}$ nuclease activity of the Taq polymerase. A byproduct of increased intrinsic Taq processivity is increased pyrophosphorolysis, which causes some peaks to lose intensity at rates that vary dramatically depending on the adjacent sequences [5]. To eliminate this problem, sequencing Taq polymerase is formulated with divalent metal cations $\left(\mathrm{Mg}^{2+} ; \mathrm{Mn}^{2+}[6]\right)$ and another thermostable enzyme named inorganic pyrophosphatase [4]. However, unevenness of peak height is also driven by the rate of incorporation of dye terminators, which is dependent on the dye type, as well as the adjacent sequences [3]. Uneven peak heights decrease the 
accuracy of base calling, and make the estimation of copy-number proportions of mixed molecular species less reliable and detection of polymorphism more difficult. Indeed, a disparity in peak heights at a polymorphic site in heterozygotes has been demonstrated, i.e., at the site of heterozygosity $\left(5^{\prime}-\mathrm{CTC}-3^{\prime} / 5^{\prime}-\mathrm{CTT}-3^{\prime}\right)$, height of the cytosine $(\mathrm{C})$ peak was 3.5-fold higher than the thymine (T) peak, whereas when using template DNA with even proportions between the two alleles, a 1:1 ratio would have been expected for this double peak [3]. Overscaled C signals and high background noise have indeed been indicated as the reasons why the approach of direct sequencing failed to gain acceptance as a reliable method for quantification of copy-number proportions from sequencing chromatograms [7]. This gave rise to numerous more complex methods, ranging from quantitative polymerase chain reaction (PCR) [8,9] to labor-intensive and time-consuming cloning-based protocols that involve cloning of PCR fragments, construction of recombinant vectors, identification of positive clones, and counting positive subclones following genotyping/DNA sequencing; or, it involves counting spots of next-generation sequencing (NGS) technologies and calculating the copy-number proportions based on depth of coverage [10]. However, each step of a complex method introduces a bias that might complicate quantification, leading researchers to rethink whether rectifying distortions in sequencing trace files and using direct sequencing for quantification would allow comparable accurate assessment of DNA copy-number proportions [7]. Bearing this approach in mind, this commentary is a review of the latest insights and developments relevant to estimating copy-number proportions based on direct Sanger sequencing.

\section{Single-Nucleotide Polymorphisms (SNPs) and Indels}

Sequencing diploid DNA to detect substitution of a single nucleotide at specific positions in the genome may be regarded as a subcase of analyzing copy-number proportions with an expected 1:1 ratio. Automated direct DNA sequencing of PCR products with BigDye-terminator chemistry has been proven to yield superior quality data compared to dRhodamine terminators and is now the most widely used approach [11]. As the pattern of dye-terminator incorporation is dependent on the local sequence context, inaccurate base calling due to uneven peak pattern remains a problem when sequencing with BigDye terminators. In the local sequence context, the two bases immediately $3^{\prime}$ to the substituted nucleotide are the most influential [3]. In most cases, their influence is similar on both alleles, allowing the 1:1 ratio to be maintained in the double peak of the heterozygosity site. However, a few cases, such as $5^{\prime}-\mathrm{YTC}-3^{\prime}$ and $5^{\prime}-\mathrm{NTT}-3^{\prime}$, result in large and small peaks at the $3^{\prime}$ base, respectively [3]. In such cases, the peak height could potentially differ 10-fold [3], dropping the T peak to background level and rendering it undetectable. Nevertheless, differential dye-terminator incorporation or preferential amplification of one allele during PCR are unlikely explanations for the complete absence of a second peak among heterozygotes, especially in cases where this absence is noted in only one of the two sequencing orientations [12]. Termed replication diode, complete absence has been shown to arise from the presence of stem-loop structures capable of guanine (G)-T wobblepairing within the tested amplicon. Stabilization of these structures for specific alleles in heterozygous situations mediates the orientation bias by hindering DNA polymerase passage on one strand, while, on the complementary strand, the non-paired adenine (A)-C nucleotide counterparts allow unobstructed replication [13]. Thus, bidirectional sequencing is mandatory for heterozygote detection. When sequencing heterozygous alleles, the different sequence context may also affect the rate at which an allele migrates in the capillary. This means that two heterozygous peaks may not always align and overlap perfectly, with one appearing a head of the other. Moreover, the fluorophores have overlapping spectra that complicate the determination of which one is present. Thus, at positions where two similar fluorophore spectral are present, it may be difficult for the sequencer hardware and software to correctly identify what is present resulting in a systemic error. Nevertheless, inferring relative proportions of DNA variants from Sanger sequencing electropherograms has gained scientific acceptance, and computer software for this purpose has been devel- 
oped. These include a free desktop application (QSVanalyzer [14], Table 1) that allows high-throughput quantification of the proportions of DNA sequences containing singlenucleotide sequence variants. A notable commercial application for such quantification is the Mutation Quantifier application of the Mutation Surveyor Software package [15]. This application improves the detection of variants with low copy-number proportion $(\sim 5 \%)$ by comparing the observed and expected peak heights, based on the assumption that intensity ratio of the neighboring same-color peaks is consistent in the samples; this is not always valid, as peak intensities are influenced by their local sequence context [3].

Microindels (1 to 50 nucleotides) are more readily detectable than SNPs, as they result in predictable superimposed trace files following the indel site [16,17]. This detectability has been harnessed to the analysis of heteroplasmic mitochondrial deletions, allowing identification of deleted molecules present in just $5 \%$ of the mixture by a specialized tool for detecting low-abundance indels in standard sequence traces [18]. Indeed, the need for techniques to estimate mitochondrial DNA copy number for human clinical diagnosis promoted advances in the detection of mitochondrial DNA heteroplasmic variations (reviewed by $[19,20])$. A method termed polymorphism-ratio sequencing (PRS) was developed for this purpose based on microfabricated capillary array electrophoresis and the Sanger protocol [21]. However, this method's limit of minor allele frequency detection was 5\%, compared to the limit of similar magnitude (5-7.5\%) reported for standard Sanger sequencing or SNaPshot minisequencing with BigDye terminators and capillary sequencers $[15,22,23]$. For indels, in base strings where the local sequence context is retained, the peak-height ratio between a reference base and its corresponding base in the molecule with the indel could potentially be used to accurately quantify their relative copy-number proportions. However, the need to quantify the efficiency of genome-editing enzymes further promoted the development of more sophisticated methods capable of sensitive analyses of indels in Sanger trace files.

\section{Base Editing by CRISPR-Cas9 Endonucleases and Nickases}

Effective tools based on Cas9 endonucleases and nickases have been developed for the purpose of gene editing with single-base resolution. These base editors rely on the riboprotein complex of Cas9 with a guide RNA to specifically localize these enzymes to the targeted site; their action results in a high frequency of base substitutions and indels [24]. Analysis of the base-editing results typically requires expensive and timeconsuming methods, such as Surveyor nuclease assay, subcloning, and NGS [25]. To overcome these limitations, several groups have recently developed computer programs to measure base-editing efficiency from Sanger sequencing trace files, including: Tracking of Indels by DEcomposition (TIDE and TIDER for easy quantification of template-directed CRISPR-Cas9 editing [26]), Edit Deconvolution by Inference of Traces in R (EditR [27]), Inference of CRISPR Edits (ICE [28]), Base-Editing Evaluation Program (BEEP [29]), and Base Editing Analysis Tool (BEAT [30]). These applications, implemented in R and Python, differ in their ability to handle indels and base substitutions (Table 1). The Python versions, developed more recently, add capacity, different statistics, and graphical output. It should be noted that although these tools specifically target analysis of CRISPR-Cas9 outcomes, their use can be easily adapted to the quantification of mixed molecular species derived from other sources, such as bisulfite sequencing. 
Table 1. Free software for estimating copy-number proportions based on Sanger sequencing.

\begin{tabular}{|c|c|c|c|c|c|}
\hline Software & Implemented in & Use Focus & Download/Tool Site & $\begin{array}{l}\text { Web } \\
\text { Tool }\end{array}$ & Reference \\
\hline QSVanalyzer & VB.Net & $\begin{array}{l}\text { Single-Nucleotide } \\
\text { Polymorphisms } \\
\text { (SNPs) }\end{array}$ & $\begin{array}{l}\text { http:/ / dna-leeds.co.uk/qsv/download.php } \\
\text { (accessed on 16 February 2021) }\end{array}$ & $\mathrm{N}$ & [14] \\
\hline BioEdit & $\mathrm{C}++$ & SNPs & $\begin{array}{l}\text { https:/ / bioedit.software.informer.com/ } \\
\text { (accessed on } 16 \text { February 2021) }\end{array}$ & $\mathrm{N}$ & [31] \\
\hline Chromas & $\mathrm{C}++$ & SNPs & $\begin{array}{l}\text { http:/ / technelysium.com.au/wp/chromas/ } \\
\text { (accessed on 16 February 2021) }\end{array}$ & $\mathrm{N}$ & Freemium \\
\hline TIDE/TIDER & $\mathrm{R}$ & CRISPR indels & $\begin{array}{l}\text { https: / / tide.nki.nl/ (accessed on } 16 \text { February } \\
\text { 2021) }\end{array}$ & $\mathrm{Y}$ & [26] \\
\hline ICE & Python & CRISPR indels & $\begin{array}{c}\text { https://ice.synthego.com/\#/ (accessed on } 16 \\
\text { February 2021) }\end{array}$ & Y & [28] \\
\hline EditR & $\mathrm{R}$ & CRISPR SNPs & $\begin{array}{c}\text { https:/ / moriaritylab.shinyapps.io/editr_v10/ } \\
\text { (accessed on 16 February 2021) }\end{array}$ & $\mathrm{Y}$ & [27] \\
\hline BEEP & Python & CRISPR indels & $\begin{array}{c}\text { https://github.com/mitmedialab/BEEP } \\
\text { (accessed on } 16 \text { February 2021) }\end{array}$ & $\mathrm{N}$ & [29] \\
\hline BEAT & Python & CRISPR SNPs & $\begin{array}{c}\text { https://hanlab.cc/beat/ (accessed on } 16 \\
\text { February 2021) }\end{array}$ & $\mathrm{Y}$ & [30] \\
\hline MultiEditR & $\mathrm{R}$ & RNA SNPs & $\begin{array}{l}\text { https:/ / moriaritylab.shinyapps.io/multieditr/ } \\
\text { (accessed on } 16 \text { February 2021) }\end{array}$ & Y & [32] \\
\hline
\end{tabular}

\section{C Methylation}

DNA methylation of $C$ occurs predominantly on $\mathrm{CpG}$ dinucleotides in eukaryotes and seldom on non-CpG bases in metazoans, including human embryonic stem and neuronal cells [33]. It has an important role in the transcriptional regulation of numerous physiological processes; thus, rapid and simple detection of DNA methylation is generating growing interest in both academic and pharmaceutical sectors [33]. Bisulfite treatment of DNA efficiently $(>99 \%)$ converts unmethylated C into uracil (U), which is amplified as T during PCR amplification. Sanger sequencing of these PCR amplicons yields chromatograms that can be utilized to calculate the methylation status of each individual $C$ by assessing the peak ratio between $C$ and $T$, representing the methylated and unmethylated $C$ in the original template DNA, respectively [7]. Using the BigDye terminator kit and a series of optimization strategies and techniques, it has been shown that the ratio between $C$ and $\mathrm{T}$ peak heights measured by Chromas software can accurately represent copy-number proportions between these bases, solving the problems of methylation quantification by direct bisulfite-PCR sequencing [7]. Yet, as the fluorophore dyes labelling $C$ and $T$ have close emission spectrum maxima (dROX $595 \mathrm{~nm}$ and dTAMRA $618 \mathrm{~nm}$, respectively), this analysis might be complicated when their respective peaks are not perfectly aligned. Better peak-height predictability in $\mathrm{CpG}$ results can be obtained if sequencing is performed from the reverse orientation, which also yields CG, but always retaining the complementary $\mathrm{C}$ preceding the methylation site as the most influential residue in the local sequence context (Table 2). As Chromas provides a "Reverse" button, sequence traces that were performed in one orientation can be presented as if obtained from the other orientation. In such a presentation, it would be expected that in the case of even proportions, the T peak will be larger than the $\mathrm{C}$ peak, and indeed up to $30 \%$ overscaling of $\mathrm{T}$ peaks has been observed for the trace chromatogram of 50\% expected values [7]. Thus, interpreting the C:T peak ratios obtained in both sequencing orientations while considering the expected C:T peak-ratio bias reported in the literature may provide a better estimation of C-methylation status. Without correcting for the peak-ratio bias, direct bisulfite sequencing is not considered reliable for quantification [34]. 
Table 2. Peak patterns in Taq-FS sequencing traces with $5^{\prime}$-NCS- $3^{\prime}$ base strings following [3].

\begin{tabular}{cccc}
\hline Base String $^{1}$ & G Peak Height & Base String $^{\mathbf{1}}$ & C Peak Height \\
\hline GCG & small & GCC & average/large \\
TCG & small & TCC & ND \\
CCG & ND & CCC & ND \\
ACG & small & ACC & average/large \\
\hline
\end{tabular}

${ }^{1}$ A string of nucleotides in which the $3^{\prime}$ base is in bold type.

\section{RNA Editing}

There are two known types of messenger RNA editing: adenosine-to-inosine (A-to-I), which is common in all animals; and cytidine-to-uridine (C-to-U), which is rare in mammals but commonly seen in plants. In reverse-transcribed PCR-amplified cDNA, these edits are visualized as A-to-G and C-to-T base substitutions, respectively [35]. Using Sanger chromatograms, determination of the proportion of A-to-I editing is based on measuring the heights of the A and $\mathrm{G}$ peaks at the edited position and then dividing the height of the $G$ peak by the sum of the $A$ and $G$ peak heights at this site. Peak heights can be measured automatically using a peak-calling program such as BioEdit or Chromas [36]. An automated tool is currently under development, based on an easy validation method for detecting and quantifying RNA editing from Sanger sequencing (Table 1, [32]). However, users are required to manually trim the $5^{\prime}$ and $3^{\prime}$ ends of the trace file to reduce noisy sequencing. Moreover, with no correction for peak-ratio bias, quantification of RNA editing from direct sequencing will be less reliable in base strings that are prone to this bias.

\section{Copy-Number Variations (CNVs)}

$\mathrm{CNV}$ are common variations in chromosomal structure that play an important role in phenotypic variation and genetic disease; SNP genotyping methods that offer independent fluorescence intensities for two alleles can be used to estimate copy-number proportions between copies of segmental duplications [37]. Thus, using Sanger sequencing (AB1) or fragment analysis (FSA) trace files, investigation of peak-height ratio of SNPs within base strings that do not induce peak-ratio bias has been reported as an accurate tool for quantifying gene copy-number proportions [38-40]. As demonstrated in Figure 1, peakcalling programs and web tools designed to quantify CRISPR-Cas9 base editing from Sanger sequencing can readily be used to estimate copy-number proportions in CNVs. These tools were tested with an original trace file (Sample4491478.ab1, Supplementary Materials) that had been used to construct the 10 gene copy model of bovine FCGR2 (CD32, Table 3 and Figure 1) [40]. Note that this specific trace file was generated from the reverse orientation and was presented as if performed from the other orientation using the GAP4 assembly program [41]. All tools yielded similar quantifications, with the BEAT tool being slightly closer to the expected values due to the different background-subtraction algorithm used (Table 3). 


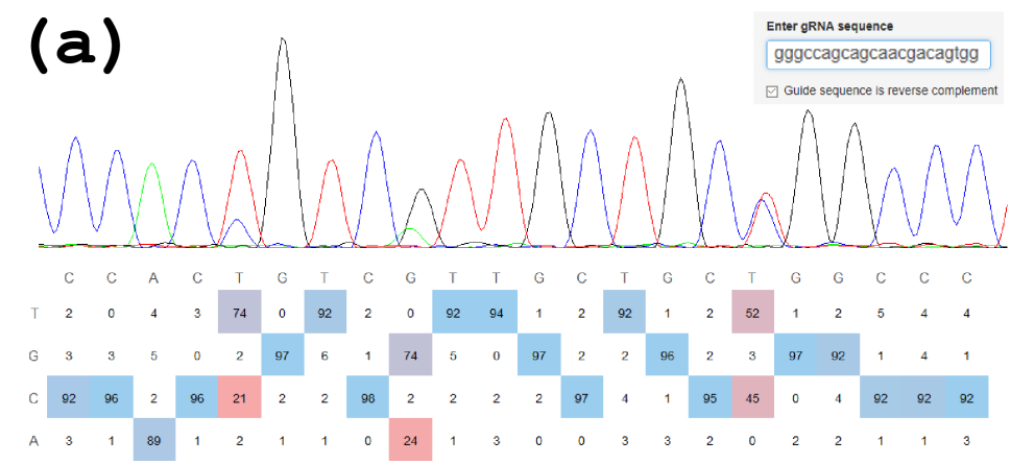

(b)
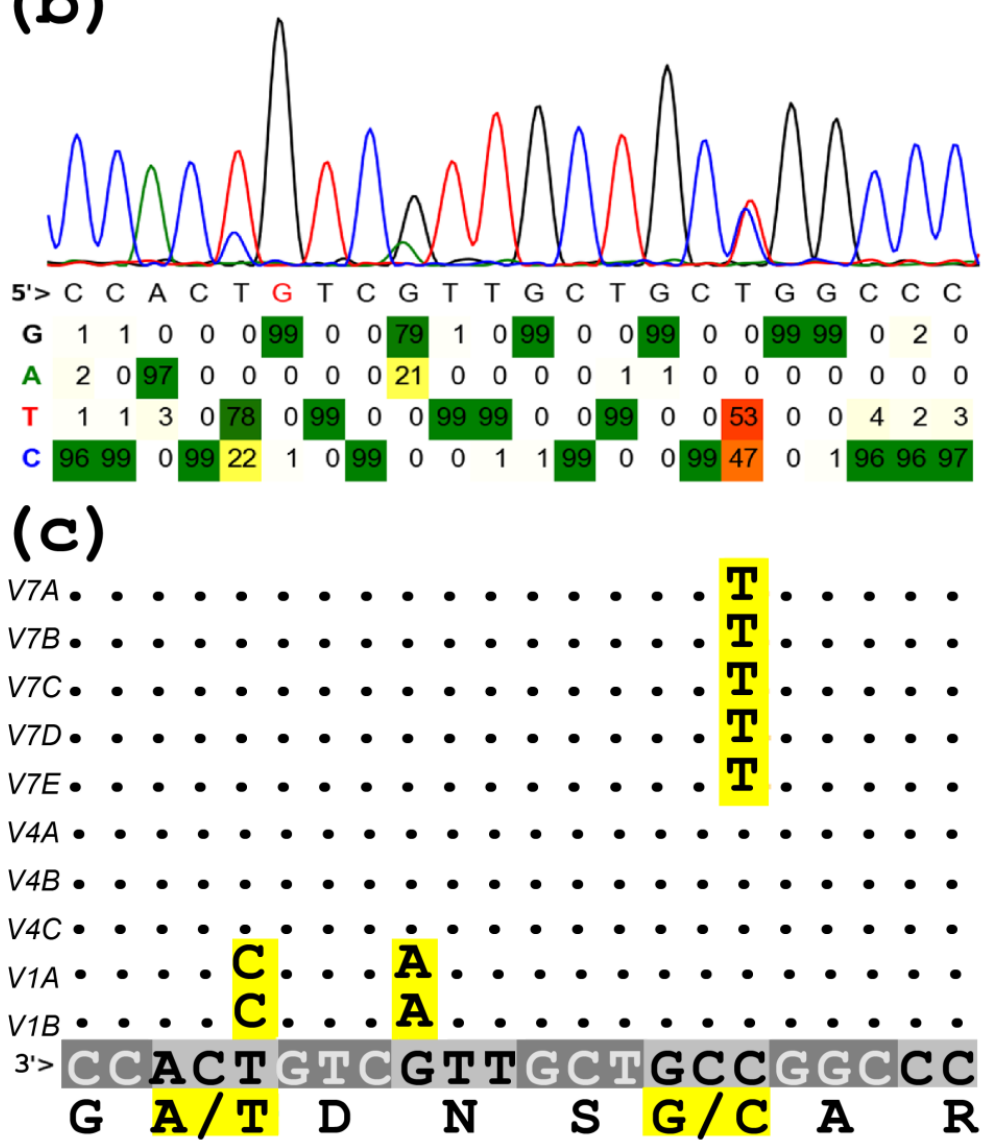

Figure 1. An example of estimating gene copy-number proportions based on sequence chromatograms of the third exon of bovine gene CD32. (a) Application of EditR, an R program for base-editing quantification [27]. (b) Application of BEAT, a Python program for base-editing quantification [30]. (c) An allele model based on DNA-sequencing that follows a previously published Figure 4 [40] but in the reverse orientation. Dots indicate similarity to the consensus sequence of 10 allele variants predicted by the assembled sequences and counts of this sire's DNA-Seq reads. Putative amino acid translation is given below the consensus sequence, in which codons are annotated by alternating font and background color. Nucleotide and amino acid variations are highlighted in yellow. The presented trace file was obtained from sequence analysis using BigDye terminators kit 3.1 (Thermo Fisher Scientific, Waltham, MA, USA) run on an ABI3730 Automated Sequencer; and analyzed by Sequencing Analysis Software 5.3.1 with KB Basecaller v1.4. 
Table 3. Estimating copy-number proportions of the bovine gene CD32 based on Sanger sequencing ${ }^{1}$.

\begin{tabular}{cccccc}
\hline Variants & Expected & EditR & BEAT & Chromas/BioEdit & QSV \\
\hline T/C & 4 & 3.52 & 3.55 & 3.50 & 3.54 \\
G/A & 4 & 3.08 & 3.79 & 3.08 & 3.03 \\
C/T & 1 & 0.87 & 0.89 & 0.87 & 0.87 \\
\hline
\end{tabular}

${ }_{1}^{1}$ The ratio between the $V 4$ type and other types is given based on the variations displayed in Figure $1 C$.

Figure 1 also demonstrates the unevenness of the peak heights resulting from the increased rate of ddGTP dye-terminator incorporation in the base string of $5^{\prime}$-CTG-3', which occurred three times in the presented sequence window. In the left instance of this base string, the $G$ peak is overscaled (170\%) compared to right-most $G$ peak (within a $5^{\prime}$-YGG-3' string). Apparently, such overscaling is not corrected for by the KB basecaller. It has been previously reported that unlike most $G$ peaks, the strength of $G$ peaks in the $5^{\prime}$-CTG-3' sequence increases disproportionately under certain reaction conditions, such as elevated concentrations of $\mathrm{Mn}^{2+}$ [6]. The recently developed basecaller PeakTrace ${ }^{\mathrm{TM}}$ (Nucleics Pty Ltd, Woollahra, Australia) is marketed as a paid enhancement of the Chromas freemium, promising better base calling and improved appearance and read length of DNA-sequencing traces [42]. However, this basecaller also does not address the adjustment of peak overscaling in base strings such as $5^{\prime}-\mathrm{CTG}-3^{\prime}$ and thus, estimation of gene copynumber proportions in CNVs using single-base variation might also be biased when using PeakTrace. This was demonstrated by an in-depth analysis of mixed base calling using mussel mitochondrial DNA in which both maternal and paternal genomes are present [43]. The demonstration compared the chromatograms generated by the KB and PeakTrace basecallers in a sequence window that included eight base variations, seven of which indicated a similar proportion between the maternal and paternal types whereas in a single occurrence at the G-to-A variation, the overscaled $(260 \%) \mathrm{G}$ peak in a $5^{\prime}$ CTG-3' string unexpectedly suggested otherwise [42]. Thus, neither basecaller corrects for this overscaling artifact. Nevertheless, such peak-height bias is readily detectable by bidirectional sequencing and therefore, analysis of gene-copy proportions based on fluorescence intensities for CNV alleles selected in nonbiased base strings may yield more accurate results compared to other complex methods, because each step of a protocol introduces its own bias, and Sanger direct sequencing has a simple protocol.

\section{Conclusions}

Recent developments in estimating copy-number proportions based on direct Sanger sequencing indicate that such quantification is gaining in scientific acceptance. Best quantification is achieved when the mixed DNA molecular species involve microindels for which superimposed trace files of the indel site allow peak-height ratio analysis. Frequent indels are associated with outcomes of CRISPR-Cas9 base editing and with analyses of mitochondrial heteroplasmy; and the need to quantify these sparked the development of new software tools to infer copy-number proportions from Sanger trace files. As the rate of incorporation of dye terminators is dependent on dye type, the adjacent sequence string and the secondary structure of the sequenced strand, such inference is less acceptable in cases of single-base variations. Frequent observation of overscaled $C$ signals renders direct bisulfite sequencing unreliable for quantification of $\mathrm{CpG}$ methylation. However, use of peak-height analysis of a single site has been reported to successfully determine gene copy-number proportions in CNVs and accurately quantify RNA editing. Thus, identifying and rectifying distortions in sequencing trace files may further promote the use of direct Sanger sequencing for quantification. Aside from consulting the available literature presenting characterized base strings associated with distortions, bidirectional sequencing is an easy way to identify and avoid these local sequence effects. Commercial companies have not disclosed the algorithms of their basecallers, which are mostly marketed as part of the software that operates capillary sequencers. However, it is apparent that despite high predictability of distorting effects on peak intensities, current basecallers focus on 
the identification of bases and not on reporting their relative proportions in mixed DNA templates. This emphasizes the need for base-calling algorithms that can take into account the effects of base terminators on incorporation rates. As different rates are generated by the ever-changing microenvironments of different salt and DNA concentrations, using sophisticated methods, such as neural networks, for base calling [44] may provide the solution for identical nucleotide resolution, which would reflect their true copy-number proportions.

Supplementary Materials: The following are available online at https://www.mdpi.com/2073-442 5/12/2/283/s1, Trace file S1: Sample4491478.ab1.

Funding: This work was supported by BARD grant number US-4997-17 from the US-Israel Binational Agricultural Research and Development (BARD) Fund.

Institutional Review Board Statement: Not applicable.

Informed Consent Statement: Not applicable.

Data Availability Statement: Please refer to suggested Data Availability Statements in section “MDPI Research Data Policies" at https:/ /www.mdpi.com/ethics (accessed on 16 February 2021).

Acknowledgments: Uri Seroussi is acknowledged for careful reading of the manuscript and helpful comments and suggestions.

Conflicts of Interest: The authors declare no conflict of interest.

\section{References}

1. Sanger, F; Coulson, A.R. A rapid method for determining sequences in DNA by primed synthesis with DNA polymerase. J. Mol. Biol. 1975, 94, 441-448. [CrossRef]

2. Ishino, S.; Ishino, Y. DNA polymerases as useful reagents for biotechnology —-The history of developmental research in the field. Front. Microbiol. 2014, 5, 465. [CrossRef] [PubMed]

3. Parker, L.T.; Zakeri, H.; Deng, Q.; Spurgeon, S.; Kwok, P.Y.; Nickerson, D.A. AmpliTaq(R) DNA polymerase, FS dye-terminator sequencing: Analysis of peak height patterns. Biotechniques 1996, 21, 694-699. [CrossRef] [PubMed]

4. Vander Horn, P.B.; Davis, M.C.; Cunniff, J.J; Ruan, C.; McArdle, B.F.; Samols, S.B.; Szasz, J.; Hu, G.; Hujer, K.M.; Domke, S.T.; et al. Thermo Sequenase DNA polymerase and T. acidophilum pyrophosphatase: New thermostable enzymes for DNA sequencing. Biotechniques 1997, 22, 758-762. [CrossRef]

5. Tabor, S.; Richardson, C.C. DNA sequence analysis with a modified bacteriophage T7 DNA polymerase. Effect of pyrophosphorolysis and metal ions. J. Biol. Chem. 1990, 265, 8322-8328. [CrossRef]

6. Korch, C.; Drabkin, H. Improved DNA sequencing accuracy and detection of heterozygous alleles using manganese citrate and different fluorescent dye terminators. Genome Res. 1999, 9, 588-595.

7. Jiang, M.; Zhang, Y.; Fei, J.; Chang, X.; Fan, W.; Qian, X.; Zhang, T.; Lu, D. Rapid quantification of DNA methylation by measuring relative peak heights in direct bisulfite-PCR sequencing traces. Lab. Investig. 2009, 90, 282-290. [CrossRef]

8. Shen, W.; Tian, Y.; Ran, T.; Gao, Z.Q. Genotyping and quantification techniques for single-nucleotide polymorphisms. Trac-Trends Anal. Chem. 2015, 69, 1-13. [CrossRef]

9. Lefever, S.; Rihani, A.; Van der Meulen, J.; Pattyn, F.; Van Maerken, T.; Van Dorpe, J.; Hellemans, J.; Vandesompele, J. Cost-effective and robust genotyping using double-mismatch allele-specific quantitative PCR. Sci. Rep. 2019, 9, 1-11. [CrossRef]

10. Roca, I.; Gonzalez-Castro, L.; Fernandez, H.; Couce, M.L.; Fernandez-Marmiesse, A. Free-access copy-number variant detection tools for targeted next-generation sequencing data. Mutat. Res. Rev. Mutat. Res. 2019, 779, 114-125. [CrossRef] [PubMed]

11. Taillon-Miller, P.; Piernot, E.E.; Kwok, P.Y. Efficient approach to unique single-nucleotide polymorphism discovery. Genome Res. 1999, 9, 499-505. [PubMed]

12. Humma, L.M.; Farmerie, W.G.; Wallace, M.R.; Johnson, J.A. Sequencing of beta 2-adrenoceptor gene PCR products using Taq BigDye terminator chemistry results in inaccurate base calling. Biotechniques 2000, 29, 962-970. [CrossRef] [PubMed]

13. Shirak, A.; Seroussi, U.; Gootwine, E.; Seroussi, E. Sequence motifs capable of forming DNA stem-loop structures act as a replication diode. FEBS Open Bio. 2017, 7, 944-952. [CrossRef]

14. Carr, I.M.; Robinson, J.I.; Dimitriou, R.; Markham, A.F.; Morgan, A.W.; Bonthron, D.T. Inferring relative proportions of DNA variants from sequencing electropherograms. Bioinformatics 2009, 25, 3244-3250. [CrossRef] [PubMed]

15. McCluskey, M.; Ni, S.; Hulce, D.; Liu, J. DNA Mutation and Methylation Quantification from Sanger Sequencing Traces with Mutation Surveyor Software. Available online: https://softgenetics.com/PDF/MutationSurveyorQuantification.pdf (accessed on 16 February 2021).

16. Seroussi, E.; Ron, M.; Kedra, D. ShiftDetector: Detection of shift mutations. Bioinformatics 2002, 18, 1137-1138. [CrossRef] [PubMed] 
17. Hill, J.T.; Demarest, B.L.; Bisgrove, B.W.; Su, Y.C.; Smith, M.; Yost, H.J. Poly peak parser: Method and software for identification of unknown indels using sanger sequencing of polymerase chain reaction products. Dev. Dyn. 2014, 243, 1632-1636. [CrossRef] [PubMed]

18. Zhidkov, I.; Cohen, R.; Geifman, N.; Mishmar, D.; Rubin, E. CHILD: A new tool for detecting low-abundance insertions and deletions in standard sequence traces. Nucleic Acids Res. 2010, 39, e47. [CrossRef]

19. Sobenin, I.A.; Mitrofanov, K.Y.; Zhelankin, A.V.; Sazonova, M.A.; Postnov, A.Y.; Revin, V.V.; Bobryshev, Y.V.; Orekhov, A.N. Quantitative assessment of heteroplasmy of mitochondrial genome: Perspectives in diagnostics and methodological pitfalls. Biomed. Res. Int. 2014, 2014, 292017. [CrossRef]

20. Duan, M.; Tu, J.; Lu, Z. Recent Advances in Detecting Mitochondrial DNA Heteroplasmic Variations. Molecules 2018, 23, 323. [CrossRef]

21. Blazej, R.G.; Paegel, B.M.; Mathies, R.A. Polymorphism ratio sequencing: A new approach for single nucleotide polymorphism discovery and genotyping. Genome. Res. 2003, 13, 287-293. [CrossRef] [PubMed]

22. Rohlin, A.; Wernersson, J.; Engwall, Y.; Wiklund, L.; Bjoerk, J.; Nordling, M. Parallel sequencing used in detection of mosaic mutations: Comparison with four diagnostic DNA screening techniques. Hum. Mutat. 2009, 30, 1012-1020. [CrossRef] [PubMed]

23. Naue, J.; Sanger, T.; Schmidt, U.; Klein, R.; Lutz-Bonengel, S. Factors affecting the detection and quantification of mitochondrial point heteroplasmy using Sanger sequencing and SNaPshot minisequencing. Int. J. Legal Med. 2011, 125, 427-436. [CrossRef]

24. Zhang, S.; Shen, J.; Li, D.; Cheng, Y. Strategies in the delivery of Cas9 ribonucleoprotein for CRISPR/Cas9 genome editing. Theranostics 2021, 11, 614-648. [CrossRef]

25. Ran, F.A.; Hsu, P.D.; Wright, J.; Agarwala, V.; Scott, D.A.; Zhang, F. Genome engineering using the CRISPR-Cas9 system. Nat. Prot. 2013, 8, 2281-2308. [CrossRef]

26. Brinkman, E.K.; Chen, T.; Amendola, M.; van Steensel, B. Easy quantitative assessment of genome editing by sequence trace decomposition. Nucleic Acids Res. 2014, 42, e168. [CrossRef]

27. Kluesner, M.G.; Nedveck, D.A.; Lahr, W.S.; Garbe, J.R.; Abrahante, J.E.; Webber, B.R.; Moriarity, B.S. EditR: A Method to quantify base editing from Sanger sequencing. CRISPR J. 2018, 1, 239-250. [CrossRef] [PubMed]

28. Hsiau, T.; Maures, T.; Waite, K.; Yang, J.; Kelso, R.; Holden, K.; Stoner, R. Inference of CRISPR edits from Sanger trace data. bioRxiv 2019, 251082.

29. Chatterjee, P.; Jakimo, N.; Jacobson, J.M. Minimal PAM specificity of a highly similar SpCas9 ortholog. Sci. Adv. 2018, 4, eaau0766. [CrossRef]

30. Xu, L.; Liu, Y.; Han, R. BEAT: A Python program to quantify base editing from Sanger sequencing. CRISPR J. 2019, 2, 223-229. [CrossRef]

31. Hall, T.A. BioEdit: A user-friendly biological sequence alignment editor and analysis program for Windows $95 / 98 / \mathrm{NT}$. Nucleic Acids Symp. Ser. 1999, 41, 95-98.

32. Kluesner, M.G.; Arnold, A.; Lerner, T.; Tasakis, R.N.; Wüst, S.; Binder, M.; Moriarity, B.S. MultiEditR: An easy validation method for detecting and quantifying RNA editing from Sanger sequencing. bioRxiv 2019, 633685.

33. Wreczycka, K.; Gosdschan, A.; Yusuf, D.; Gruning, B.; Assenov, Y.; Akalin, A. Strategies for analyzing bisulfite sequencing data. J. Biotechnol. 2017, 261, 105-115. [CrossRef] [PubMed]

34. Pajares, M.J.; Palanca-Ballester, C.; Urtasun, R.; Alemany-Cosme, E.; Lahoz, A.; Sandoval, J. Methods for analysis of specific DNA methylation status. Methods 2020, in press.

35. Toung, J.M.; Lahens, N.; Hogenesch, J.B.; Grant, G. Detection theory in identification of RNA-DNA sequence differences using RNA-sequencing. PLoS ONE 2014, 9, e112040. [CrossRef]

36. Oakes, E.; Vadlamani, P.; Hundley, H.A. Methods for the detection of adenosine-to-inosine editing events in cellular RNA. In mRNA Processing: Methods and Protocols; Shi, Y., Ed.; Springer: New York, NY, USA, 2017; pp. 103-127.

37. Feuk, L.; Carson, A.R.; Scherer, S.W. Structural variation in the human genome. Nat. Rev. Genet. 2006, 7, 85-97. [CrossRef]

38. Seroussi, E.; Klompus, S.; Silanikove, M.; Krifucks, O.; Shapiro, F.; Gertler, A.; Leitner, G. Nonbactericidal secreted phospholipase A2s are potential anti-inflammatory factors in the mammary gland. Immunogenetics 2013, 65, 861-871. [CrossRef] [PubMed]

39. Curzon, A.Y.; Shirak, A.; Dor, L.; Zak, T.; Perelberg, A.; Seroussi, E.; Ron, M. A duplication of the Anti-Mullerian hormone gene is associated with genetic sex determination of different Oreochromis niloticus strains. Heredity (Edinb) 2020, 125, 317-327. [CrossRef]

40. Seroussi, E.; Blum, S.E.; Krifucks, O.; Shirak, A.; Jacoby, S.; Leitner, G. Basal levels of CD18 antigen presenting cells in cow milk associate with copy-number variation of Fc Gamma Receptors. Genes (Basel) 2020, 11, 952. [CrossRef]

41. Staden, R.; Beal, K.F.; Bonfield, J.K. The Staden package, 1998. Methods Mol. Biol. 2000, 132, 115-130.

42. Tillett, D. In Depth: Mixed Basecalling and Simple Mixed Basecalling. Available online: http://cowry.agri.huji.ac.il/ InDepthMixedBasecalling.htm (accessed on 16 February 2021).

43. Zouros, E. Biparental Inheritance through uniparental transmission: The doubly uniparental inheritance (DUI) of mitochondrial DNA. Evol. Biol. 2013, 40,1-31. [CrossRef]

44. Allex, C.F.; Shavlik, J.W.; Blattner, F.R. Neural network input representations that produce accurate consensus sequences from DNA fragment assemblies. Bioinformatics 1999, 15, 723-728. [CrossRef] 